\title{
Reentrance of Bose-Einstein condensation in spinor atomic gases in magnetic field
}

\author{
Chengjun Tao, Zhirong Jiao, and Qiang Gu* \\ Department of Physics, University of Science and Technology Beijing, Beijing 100083, People's Republic of China
}

(Dated: November 20, 2018)

\begin{abstract}
We calculate the Bose-Einstein condensation (BEC) temperature of spin-1 atomic bosons in external magnetic field, taking into account the influence of the quadratic Zeeman effect. In case that the quadratic Zeeman coefficient is positive, the BEC temperature exhibits a nontrivial dependance on the magnetic field and a magnetic-field-induced reentrant phenomenon of BEC is observed. This phenomenon could be well understood by the competition between the linear and quadratic Zeeman effects. Reentrance of BEC in a trapped spinor Bose gas is also discussed.
\end{abstract}

PACS numbers: 03.75.Mn, 03.75.Hh, 05.30.Jp, 75.30.Kz

\section{INTRODUCTION}

Since the first observation of spin- ${ }^{23} \mathrm{Na}$ Bose-Einstein condensation (BEC) in the optical trap [1, 2], Bose gas with spin degrees of freedom, called the spinor Bose gas [3, 4], becomes one of the central topics in cold atomic physics. The spin degrees of freedom permit a variety of additional exotic phenomena to be manifest. Considerable experimental and theoretical works have been dedicated to investigating static or dynamic properties relevant to spins of the condensate, including magnetic ordering [3, 4, 5, 5] , coherent spin-dynamics 7, 8, 9, 10, 11], spin-domains or textures [12, 13, 14, 15, 16, 17] and so on. In addition, a number of researchers have laid eyes on finite-temperature properties of spinor bosons, especially on the BEC phenomenon itself [18, 19, 20, 21, 22, 23].

Bose-Einstein condensation in a free spin-1 Bose gas has ever been studied by Yamada [18], Simkin and Cohen [19]. The obtained results suggest that the Bose gas is more sensitive to external magnetic field than a Fermi gas and the BEC temperature rises up with the magnetic field due to the Zeeman effect. If there are ferromagnetic (FM) couplings between atoms, BEC occurs in a more fascinating way because the FM coupling induces a new phase transition, the FM transition [20]. Its critical temperature is called the Curie point. It is already demonstrated that the Curie point is never below the BEC temperature and both critical points are increased by the FM coupling [20]. These conclusions have also been confirmed by other groups [21]. Moreover, Bose-Einstein condensation in an optically trapped spinor Bose gas has been elaborately studied [22, 23].

In the present study, we concentrate on the BoseEinstein condensation in an $F=1$ spinor Bose gas by considering quadratic Zeeman effect (QZE). As Stenger et al. indicated [2], the Zeeman energy of a spin-1 Bose atom is given by

$$
E_{z e}^{\sigma}=E_{0}-p h \sigma+q h^{2} \sigma^{2},
$$

where $\sigma$ refers to the spin- $z$ index of hyperfine state

*Electronic address: qgu@sas.ustb.edu.cn
$\left|F=1, m_{F}=\sigma\right\rangle(\sigma=+1,0,-1), h$ to the magnetic field, and $E_{0}$ to the Zeeman energy of the $m_{F}=0$ state. The second term represents the linear Zeeman splitting and the last term arises from the quadratic Zeeman effect. For a spinor atom, the quadratic Zeeman effect can not be neglected due to the hyperfine structure 24, 25]. Very recently it is found that the QZE can even be induced by the dipole trap, which is used to confine cold atoms experimentally, for the spinor atom ${ }^{52} \mathrm{Cr}$ [25].

The QZE plays an important role for the understanding of spinor atomic bosons, especially in strong magnetic field. Various of effects caused by the quadratic Zeeman shift have been reported in recent years. It, together with the linear Zeeman effect and spin-dependant interactions, can not only lead to a number of novel ground states 2, 25], but also affects significantly on spin dynamics 7, 8] of the spinor condensates. The spin dynamics is dramatically suppressed in magnetic field, owing to the quadratic Zeeman splitting. The QZE can also influence on the vortex state, and bring about new type vortex states in spinor condensates [26]. In this paper, we aim at discussing the Bose-Einstein condensation of the spin1 Bose gas. Interestingly, we show that the QZE can induce reentrance of $\mathrm{BEC}$ in the Bose gas.

The spin-1 Bose gas is described by the following Hamiltonian:

$$
\begin{aligned}
\mathcal{H}= & \int d \mathbf{r}\left[\frac{\hbar^{2}}{2 m^{*}} \nabla \psi_{a}^{\dagger} \cdot \nabla \psi_{a}+\frac{c_{0}}{2} \psi_{a}^{\dagger} \psi_{b}^{\dagger} \psi_{b} \psi_{a}\right. \\
& +\frac{c_{2}}{2} \psi_{a}^{\dagger} \psi_{a^{\prime}}^{\dagger} \mathbf{F}_{a b} \cdot \mathbf{F}_{a^{\prime} b^{\prime}} \psi_{b^{\prime}} \psi_{b} \\
& \left.-p h \psi_{a}^{\dagger} F_{a b}^{z} \psi_{b}+q h^{2} \psi_{a}^{\dagger}\left(F_{a b}^{z}\right)^{2} \psi_{b}\right]
\end{aligned}
$$

where $\psi_{a}(\mathbf{r})$ is the field annihilation operator for an atom in hyperfine state $|1, a\rangle$ at point $\mathbf{r}$. The first term represents kinetic energy. The terms with coefficients $c_{0}$ and $c_{2}$ denote the spin-independent and spin-dependent interactions, respectively. The forth and fifth terms describe the linear and quadratic Zeeman effects, respectively. We suppose that the linear Zeeman coefficient $p$ is always positive hereinafter, but the quadratic Zeeman coefficient $q$ can be either positive or negative. $\mathbf{F}$ is a vector which consists of three components of the $3 \times 3$ Pauli spin-1 matrices [3, 4]. 
This paper is organized as follows. In Sec. II, we calculate the condensation temperature and the condensate fraction of homogenous spinor gases in the external magnetic field. The reentrance phenomenon is observed in case of $q>0$ and a detail explanation is given on how it is induced by the quadratic Zeeman effect in a free gas. The Sec. III extends the study to the trapped interacting gases. The interaction between atoms is treated by using a mean-field approach. It is suggested that the interaction tends to decrease the condensation temperature, but the reentrance phenomenon sustains. The last section gives a brief summary.

\section{REENTRANCE IN A HOMOGENOUS GAS}

First we consider a free spin-1 Bose gas, ignoring the interactions in the Hamiltonian (2). Then the effective Hamiltonian for the grand canonical ensemble reads

$$
\hat{H}-\hat{N} \mu=\sum_{k \sigma}\left(\varepsilon_{k}-\mu-p h \sigma+q h^{2} \sigma^{2}\right) \hat{n}_{k \sigma},
$$

where $\varepsilon_{k}=\hbar^{2} k^{2} / 2 m^{*}$ is the kinetic energy of free particles with mass $m^{*}, \mu$ is the chemical potential, and $\hat{N}$ is the operator of total particle number. Since the Hamiltonian is diagonal, we may calculate the grand thermodynamical potential

$$
\Omega=-\frac{1}{\beta} \ln Z=-\frac{1}{\beta} \ln \operatorname{Tr}\left[e^{-\beta(\hat{H}-\hat{N} \mu)}\right],
$$

where $Z=\operatorname{Tr}\left[e^{-\beta(\hat{H}-\hat{N} \mu)}\right]$ is the partition function, and $\beta=1 / k_{B} T$. The density of particles is derived as

$$
\bar{n}=-\frac{1}{V}\left(\frac{\partial \Omega}{\partial \mu_{\sigma}}\right)_{T, V}=\frac{1}{V} \sum_{k \sigma} n_{k \sigma},
$$

where $V$ is the volume of the system, and $\bar{n}=N / V$ is the particle density. From Eq. (5), we can obtain the basic equation determining the phase diagram of the spin-1 Bose system,

$$
\begin{aligned}
1= & n_{c}+\left(\frac{m^{*} k_{B} T}{2 \pi \hbar^{2} \bar{n}^{2 / 3}}\right)^{3 / 2}\left[f_{3 / 2}\left(-\frac{\mu+p h-q h^{2}}{k_{B} T}\right)\right. \\
& \left.+f_{3 / 2}\left(-\frac{\mu}{k_{B} T}\right)+f_{3 / 2}\left(-\frac{\mu-p h-q h^{2}}{k_{B} T}\right)\right],
\end{aligned}
$$

where $n_{c}=\bar{n}_{c} / \bar{n}$ is the condensate fraction, $\bar{n}_{c}$ is the condensate density, and $f_{s}(x)$ is the polylogarithm function defined as [20]

$$
f_{s}(x) \equiv L i_{s}\left(e^{-x}\right)=\sum_{k=1}^{\infty} \frac{\left(e^{-x}\right)^{k}}{k^{s}}, x \geq 0 .
$$

Above the condensation temperature $T_{c}$, the condensate fraction $n_{c}=0$ and the chemical potential $\mu$ is determined by Eq. (6) for each given temperature. At $T_{c}$,

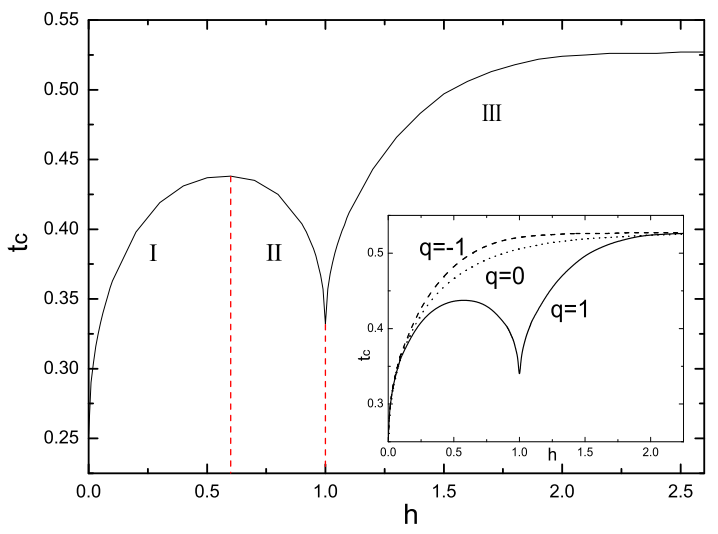

FIG. 1: Reduced BEC temperature $t_{c}$ vs external magnetic field $h$ for the spin-1 Bose gas with $q=1$. The inset plots BEC temperatures for all the three cases of $q=-1,0$ and 1 so as to make a comparison.

atoms lying on the lowest energy sub-level begin to condense. In order to determine $T_{c}$, one have to make it clear which sub-level is the lowest first. From Eq. (3) it is easy to see that the energy level for the $m_{F}=1$ state is lower than the other two as long as the external magnetic field $h$ is sufficiently weak, so the $m_{F}=1$ atoms condense once the temperature is below $T_{c}$. The chemical potential $\mu$ satisfies the equation $\mu+p h-q h^{2}<0$ above $T_{c}$, and $\mu+p h-q h^{2}=0$ at and below $T_{c}$. Then the condensate fraction is calculated by the following equation

$$
\begin{aligned}
1= & n_{c}+\left(\frac{T}{T_{c}^{0}}\right)^{3 / 2} \frac{1}{3 \zeta(3 / 2)}[\zeta(3 / 2) \\
& \left.+f_{3 / 2}\left(\frac{p h-q h^{2}}{k_{B} T}\right)+f_{3 / 2}\left(\frac{2 p h}{k_{B} T}\right)\right],
\end{aligned}
$$

where

$$
T_{c}^{0}=\frac{2 \pi \hbar^{2}}{m^{*} k_{B}}\left(\frac{\bar{n}}{3 \zeta(3 / 2)}\right)^{2 / 3}
$$

is the BEC temperature for a free spin-1 Bose gas without external field and $\zeta(3 / 2)=f_{3 / 2}(0) \approx 2.612$ is the Riemann zeta function.

As the external magnetic field becomes stronger, the energy level structure may be modified because of the influence of the QZE, especially in the case of $q>0$. When $q h^{2}>p h$, the $m_{F}=0$ sub-level becomes lower than the $m_{F}=1$ sub-level, and $m_{F}=0$ atoms condense in place of $m_{F}=1$ atoms correspondingly. Therefore, $\mu \rightarrow 0$ as $T \rightarrow T_{c}$. Below $T_{c}$, Eq. (6) reduces to

$$
\begin{aligned}
1= & n_{c}+\left(\frac{T}{T_{c}^{0}}\right)^{3 / 2} \frac{1}{3 \zeta(3 / 2)}\left[f_{3 / 2}\left(-\frac{p h-q h^{2}}{k_{B} T}\right)\right. \\
& \left.+\zeta(3 / 2)+f_{3 / 2}\left(\frac{p h+q h^{2}}{k_{B} T}\right)\right] .
\end{aligned}
$$

Based on above discussions, we produce the $t_{c}$ - $h$ phase diagram numerically, as plotted in Fig. 11. In our calculations, a reduced temperature $t=T /\left[T_{c}^{0}(3 \zeta(3 / 2))^{2 / 3}\right]=$ 


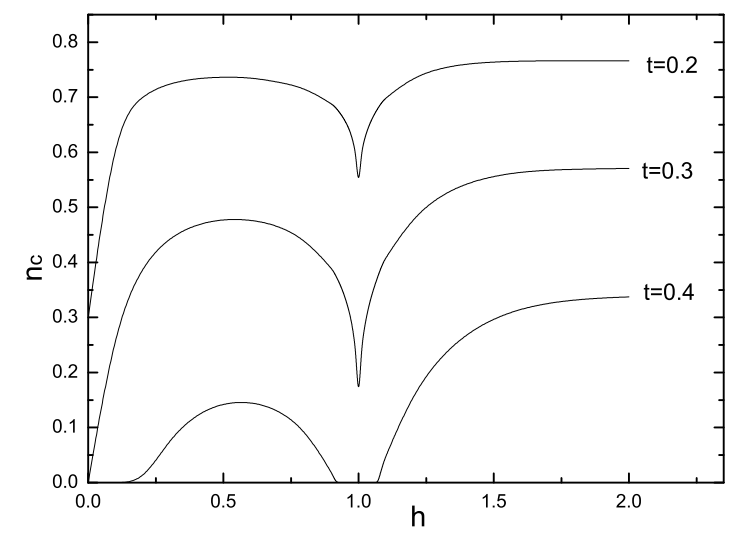

FIG. 2: Condensate fraction $n_{c}$ vs external magnetic field $h$ at different temperatures $t=0.2,0.3,0.4$.

$\left(m^{*} k_{B} T\right) /\left(2 \pi \hbar^{2} \bar{n}^{2 / 3}\right)$ is introduced, and then $p h$ and $q h^{2}$ must be re-scaled by the factor of $k_{B} T_{c}^{0}(3 \zeta(3 / 2))^{2 / 3}$. The particle density is suppose to be $1 ; p, q$ and $h$ are chosen to be dimensionless parameters and by setting the re-scale factor to be 1 . For simplicity, we fix the value of $p$ as $p=1$ and place emphasis on discussing three distinct cases with respect to the QZE, $q=-1,0$, and 1 . As shown in the inset of Fig. 10 the phase diagram for the $q=-1$ or 0 case is very simple. The BEC temperature $t_{c}$ grows monotonously as $h$ increases. In contrast, the $q=1$ case is very intriguing: $t_{c}$ increase with $h$ first, and then decreases. After dropping to a minimum value at $h=1.0$, it grows up again. During the temperature range of $t \sim 0.33$ to $t \sim 0.43$, a typical reentrant phenomenon of BEC induced by the magnetic field $h$ is observed.

To further demonstrate the reentrant phenomenon in spin- 1 atomic bosons with $q=1$, we calculate the condensate fraction $n_{c}$ at some given temperatures, as shown in Fig. 2. The condensed particles become dramatically less around $h=1.0$, corresponding to the minimum point of $t_{c}$ in Fig. 1. Especially at $t=0.4$, the condensate vanishes around $h=1.0$, and then reappears in stronger field. The reentrant phenomenon can be interpreted as the consequence of the competition between linear and quadratic Zeeman effects, as discussed in the following.

Without magnetic field, the energy spectra for the three hyperfine spin states, $E_{k}^{\sigma}=\varepsilon_{k}-\mu-p h \sigma+q h^{2} \sigma^{2}$, are degenerate. Therefore, each spin state is equally occupied, $\bar{n}_{1}=\bar{n}_{0}=\bar{n}_{-1}=\bar{n} / 3 . \bar{n} / 3$ is just the critical particle density, which determines the BEC temperature to be $t_{c}^{0}=\{1 /[3 \zeta(3 / 2)]\}^{2 / 3} \approx 0.2535$. When external magnetic field is applied, hyperfine energy levels are shifted due to both the linear and quadratic Zeeman effects. Fig. 3 shows schematically the splitting of energy levels for atoms with positive quadratic Zeeman coefficient, $q>0$. The energy dispersion with respect to $k$ is not depicted explicitly.

When $h$ is relatively week, the linear Zeeman effect dominates over the QZE and the energy level splitting is shown in Fig. 3(a), which satisfies $E_{k}^{1}<E_{k}^{0}<E_{k}^{-1}$. a)

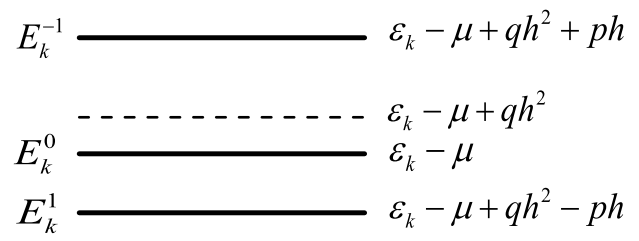

b)

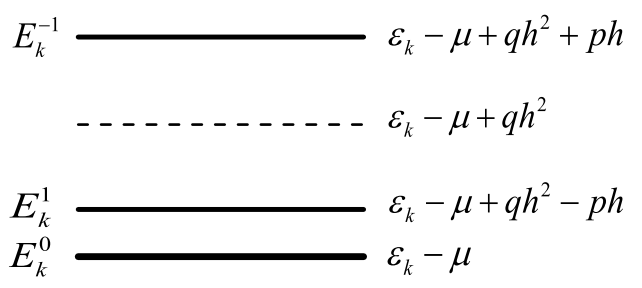

FIG. 3: Energy splittings of the three spin components in case of $q>0$ with $h$ being relatively weak (a) or strong (b).

Consequently, $\bar{n}_{1}>\bar{n}_{0}>\bar{n}_{-1}$, and $\bar{n}_{1}$ acts as the critical density of particles. Because $\bar{n}_{1}>\bar{n} / 3$, the BEC temperature is larger than $t_{c}^{0}$. As $h$ increases gradually, the three energy levels become more separated so that $\bar{n}_{1}$ and thus $t_{c}$ tend to growing. This region of $h$ is labeled as I in Fig. 11. When $h=p /(2 q)=1 / 2$, the separation between $E_{k}^{1}$ and $E_{k}^{0}$ arrives at the largest value, $p^{2} /(4 q)$. However, Fig. 1 1 shows that Region I terminates at a field $h_{1}^{*}$ larger than $1 / 2$. During $1 / 2<h<h_{1}^{*}$, though the separation between $E_{k}^{1}$ and $E_{k}^{0}$ becomes shrinking, the separation between $E_{k}^{1}\left(E_{k}^{0}\right)$ and $E_{k}^{-1}$ is still enlarged. As a result, more atoms occupy the $m_{F}=0$ and $m_{F}=1$ so that $\bar{n}_{1}$ keeps increasing with $h$, until $h=h_{1}^{*}$.

In Region II of Fig. 1, the quadratic Zeeman term $q h^{2}$ becomes dominating, with the result that $E_{k}^{0}$ becomes closer to the $E_{k}^{1}$ significantly. Although $E_{k}^{1}$ is still the lowest energy level, the occupation number $\bar{n}_{1}$ begins to decrease and $t_{c}$ drops accordingly. But otherwise, $\bar{n}_{0}$ increases. Since $E_{k}^{-1}$ is far higher than $E_{k}^{0}$ and $E_{k}^{1}$, few atoms occupy the $m_{F}=-1$ energy level. When $h$ approaches a particular value, $h_{2}^{*}=p / q=1, E_{k}^{0}=E_{k}^{1}$ and hence $\bar{n}_{0}=\bar{n}_{1}$ which is slightly smaller than $\bar{n} / 2$. So the BEC temperature $t_{c} \approx 0.3327$ is slightly higher than $(3 / 2)^{2 / 3} t_{c}^{0} \approx 0.3321$.

Increasing $h$ further, $E_{k}^{0}$ becomes the lowest energy level in place of $E_{k}^{1}$, as indicated in Fig. 3(b). Therefore the condensed atoms are in the $m_{F}=0$ state, instead of in the $m_{F}=1$ state. Meanwhile the BEC temperature $t_{c}$ grows up again. As the stronger $h$ makes the three energy levels separate farther and farther, $\bar{n}_{0} \rightarrow \bar{n}$ and $t_{c} \rightarrow 3^{2 / 3} t_{0}$ in the high field limit. This point is noted in Region III of Fig. [1.

In case of $q=0$ or $q=-1, E_{k}^{1}$ remains the lowest energy level all the way in magnetic field and only the $m_{F}=1$ atoms can condense. The magnetic field $h$ persists in enhancing splittings of the three energy levels. Correspondingly, the BEC temperature grows monotonously with $h$ tending to stronger, as shown in the inset of Fig. 1. 


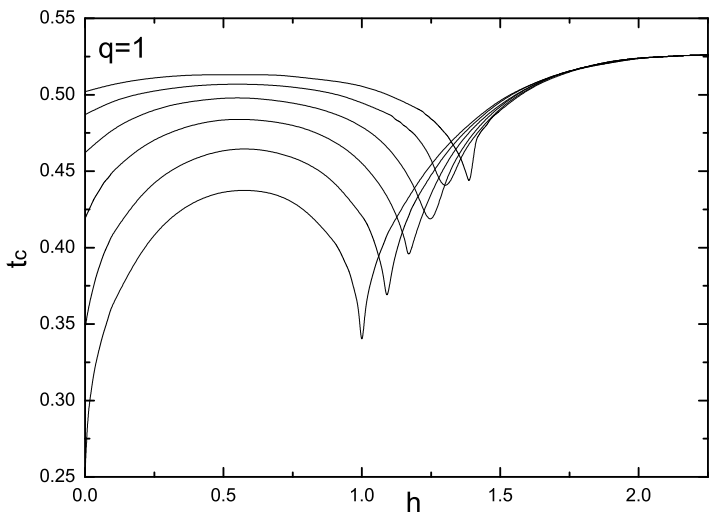

FIG. 4: Reduced BEC temperature $t_{c}$ vs external magnetic field $h$ for a ferromagnetically coupled spin-1 Bose gas. The coupling $\left|c_{2}\right|=0,0.2,0.4,0.6,0.8$, and 1 from bottom to top.

We note that the reentrant phenomenon has been discovered in a number of quantum many-particle systems, especially in some unconventional superconductors. For example, it is reported that there appear successively two distinct superconducting phases with the pressure in heavy fermion material $\mathrm{CeCu}_{2} \mathrm{Si}_{2}$ [27]. A magneticfield induced reentrance of superconductivity has ever been observed in a pseudo-ternary Eu-Sn material 28]. For cold atoms, Kleinert et al. have discussed reentrance in the quantum phase transitions of an interacting Bose gas [29]. They suggested that $T_{c}$ shifts upward with the interacting strength first, then it is suppressed to zero.

The ${ }^{87} \mathrm{Rb}$ gas happens to exhibit a positive QZE [14] and therefore it may be an appropriate example where the reentrant phenomenon is expected to be observed. There exist weak FM couplings between ${ }^{87} \mathrm{Rb}$ atoms, as described by the $c_{2}$ term in Eq. (2) with $c_{2}<0$. So we proceed to examine how the FM coupling affects on the reentrance of $\mathrm{BEC}$, by decoupling the $c_{2}$ term via mean-field theory [20],

$$
H_{c_{2}}=-\left|c_{2}\right| m \sum_{k}\left(\hat{n}_{k 1}-\hat{n}_{k-1}\right),
$$

where $m$ is the normalized magnetization defined as $m=$ $\sum_{k}\left\langle\hat{n}_{k 1}-\hat{n}_{k-1}\right\rangle / N$. Below the BEC temperature, it is given by

$$
\begin{aligned}
m= & \left(\frac{T}{T_{c}^{0}}\right)^{3 / 2} \frac{1}{3 \zeta(3 / 2)}\left[\zeta(3 / 2)-f_{3 / 2}\left(\frac{2 p h+2\left|c_{2}\right| m}{k_{B} T}\right)\right] \\
& +n_{c}
\end{aligned}
$$

if condensed atoms are in the $m_{F}=1$ state or

$$
\begin{aligned}
m= & \left(\frac{T}{T_{c}^{0}}\right)^{3 / 2} \frac{1}{3 \zeta(3 / 2)}\left[f_{3 / 2}\left(-\frac{p h+\left|c_{2}\right| m-q h^{2}}{k_{B} T}\right)\right. \\
& \left.-f_{3 / 2}\left(\frac{p h+\left|c_{2}\right| m+q h^{2}}{k_{B} T}\right)\right]
\end{aligned}
$$

if condensed atoms in the $m_{F}=0$ state.
Based on Eq. (10), when the $c_{2}$ term is included, Eqs. (6), (8) and (9) should be rewritten by replacing $p h$ with $p h+\left|c_{2}\right| m$. Hence it seems that the FM coupling performs a role to enhance the linear Zeeman effect. Combining Eqs. (8) and (11), or (9) and (12), we obtain that the reentrant phenomenon still manifests, as seen in Fig. 4. The FM coupling tends to stabilize the $m_{F}=1$ condensate, so that the BEC temperature is upraised in the weak field region of $h<h_{2}^{*}$ with the FM coupling increasing. Meanwhile, $h_{2}^{*}$ moves to the high-field side.

\section{REENTRANCE IN A TRAPPED INTERACTING BOSE GAS}

In experiments, atomic gases are usually confined in an effective three-dimensional harmonic trap, so it is useful to extend the above calculations to the trapped case. In this section, we consider a system of interacting atoms trapped by the following potential

$$
V(\mathbf{r})=\frac{1}{2} m^{*} \sum_{i} \omega_{i}^{2} r_{i}^{2}
$$

where $\omega_{i}(i=x, y, z)$ denotes the angular frequency.

There are two interaction terms in the Hamiltonian (2). Actually, the spin-dependent interaction is rather weak in comparison with the spin-independent interaction in cold atoms. For example, in the ${ }^{87} \mathrm{Rb}$ gas $c_{2}$ is about 2 orders of magnitude smaller than $c_{0}$. Therefore, in the following calculations we neglect the $c_{2}$ term and turn to deal with the $c_{0}$ term in some detail.

For a trapped scalar Bose gas, the interaction can be well-treated by the mean-field theory. The obtained results on the BEC temperature and the condensate fraction are quite accurate [30, 31]. The BEC temperature is shifted to lower temperatures in the presence of repulsive interactions [30]. Hereinafter, we calculate the BEC temperature and condensate fraction for spinor Bose gases in the magnetic field based on a generalized mean-field approach from the scalar to the spinor Bose gases [23].

According to the mean-field theory [31], the field operator could be treated as $\psi_{\sigma}=\phi_{\sigma}+\delta \psi_{\sigma}$ with $\phi_{\sigma}=$ $\left\langle\psi_{\sigma}\right\rangle$. Within the Hartree-Fock (HF) approximation, the condensate can be described by the finite-temperature generalization of the spinor Gross-Pitaevskii equations (GPEs) 23], which are given by

$$
\begin{aligned}
i \hbar \frac{\partial}{\partial t} \phi_{\sigma} & =\left(-\frac{\hbar^{2}}{2 m^{*}} \nabla^{2}+V(\mathbf{r})\right. \\
& \left.-p h \sigma+q h^{2} \sigma^{2}+c_{0}\left(n+n_{\sigma}^{T}\right)\right) \phi_{\sigma}
\end{aligned}
$$

for the $m_{F}=\sigma= \pm 1$ condensation and

$$
i \hbar \frac{\partial}{\partial t} \phi_{0}=\left(-\frac{\hbar^{2}}{2 m^{*}} \nabla^{2}+V(\mathbf{r})+c_{0}\left(n+n_{0}^{T}\right)\right) \phi_{0}
$$

for the $m_{F}=0$ condensation. Here $n_{\sigma}^{c}=\left|\phi_{\sigma}\right|^{2}, n_{\sigma}^{T}=$ $\left\langle\delta \psi_{\sigma}^{\dagger} \delta \psi_{\sigma}\right\rangle$ and $n_{\sigma}=n_{\sigma}^{T}+n_{\sigma}^{c}$ describe the condensed, noncondensed and total density of the $m_{F}=\sigma$ component, 


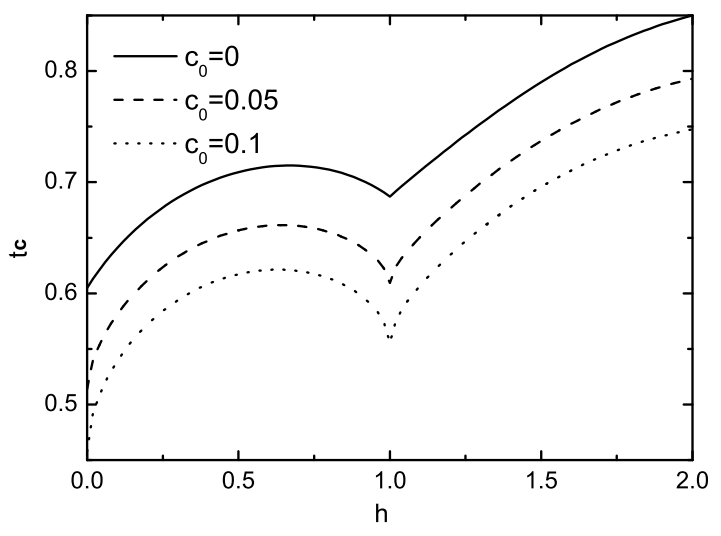

FIG. 5: Reduced BEC temperature $t_{c}$ vs external magnetic field $h$ for different interaction strength. The rescaled interaction parameter $c_{0}^{*}=0,0.05$, and 0.1 respectively.

respectively. $n=\sum_{\sigma} n_{\sigma}$ denotes the total density of all atoms. As discussed in Sec. II, the $m_{F}=-1$ atoms will never condense in the cases we considered. So for Eq. (13), we only discuss $\sigma=1$ case.

The dynamics of noncondensed atoms satisfy the following equations,

$$
\begin{aligned}
i \hbar \frac{\partial}{\partial t} \delta \psi_{1} & =\left(-\frac{\hbar^{2}}{2 m^{*}} \nabla^{2}+V(\mathbf{r})\right. \\
& \left.-p h+q h^{2}+c_{0}\left(n+n_{1}\right)\right) \delta \psi_{1} \\
i \hbar \frac{\partial}{\partial t} \delta \psi_{0} & =\left(-\frac{\hbar^{2}}{2 m^{*}} \nabla^{2}+V(\mathbf{r})+c_{0}\left(n+n_{0}\right)\right) \delta \psi_{0} \\
i \hbar \frac{\partial}{\partial t} \delta \psi_{-1} & =\left(-\frac{\hbar^{2}}{2 m^{*}} \nabla^{2}+V(\mathbf{r})\right. \\
& \left.+p h+q h^{2}+c_{0}\left(n+n_{-1}\right)\right) \delta \psi_{-1}
\end{aligned}
$$

Then we derive the effective energy spectra for all the three components,

$$
\begin{aligned}
\varepsilon_{1}(\mathbf{k}, \mathbf{r}) & =\frac{\hbar^{2} k^{2}}{2 m^{*}}+V(\mathbf{r})-p h+q h^{2}+c_{0}\left(n+n_{1}\right), \\
\varepsilon_{0}(\mathbf{k}, \mathbf{r}) & =\frac{\hbar^{2} k^{2}}{2 m^{*}}+V(\mathbf{r})+c_{0}\left(n+n_{0}\right), \\
\varepsilon_{-1}(\mathbf{k}, \mathbf{r}) & =\frac{\hbar^{2} k^{2}}{2 m^{*}}+V(\mathbf{r})+p h+q h^{2}+c_{0}\left(n+n_{-1}\right) .
\end{aligned}
$$

Using the semiclassical approximation, the density of thermal atoms with $m_{F}=\sigma$ becomes

$$
n_{\sigma}^{T}(\mathbf{r})=\int d^{3} \mathbf{k}\left[\exp \left(\frac{\varepsilon_{\sigma}(k, \mathbf{r})-\mu}{k_{B} T}\right)-1\right]^{-1},
$$

where the chemical potential $\mu$ could be derived from Eq. (13) or (14). On the basis of the Thomas-Fermi approximation, one gets that

$$
\mu=V(\mathbf{r})-p h+q h^{2}+c_{0}\left(n+n_{1}^{T}\right)
$$

when the linear Zeeman effect dominates and only the $m_{F}=\sigma=1$ atoms can condense, or

$$
\mu=V(\mathbf{r})+c_{0}\left(n+n_{0}^{T}\right)
$$
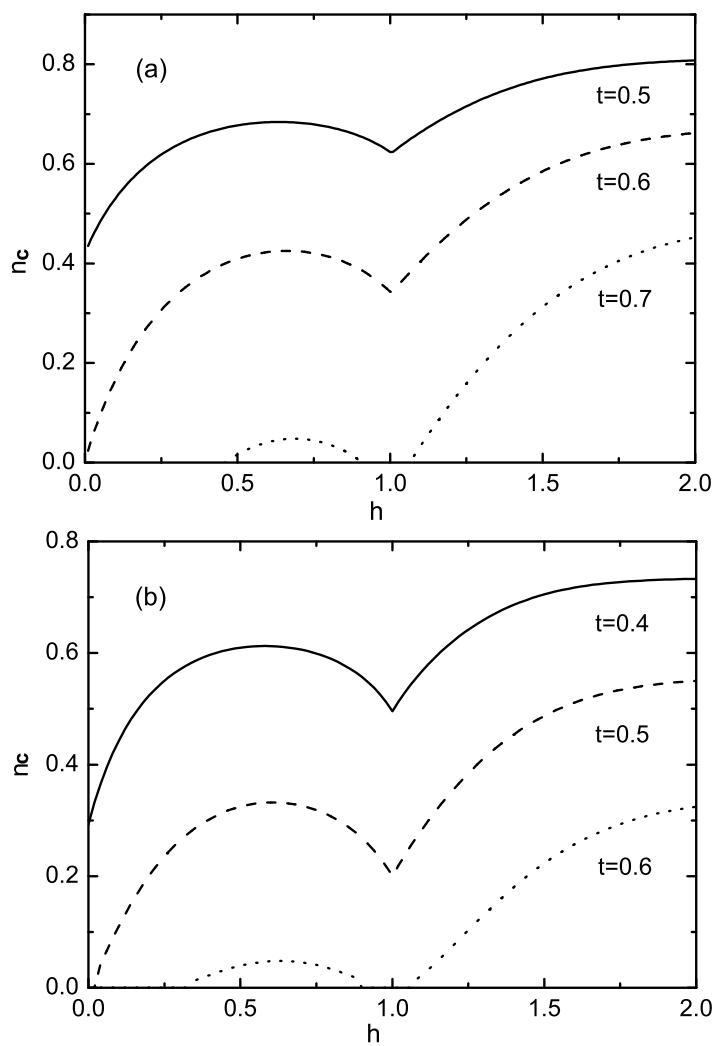

FIG. 6: (a) Condensate fraction of noninteracting Bose gas in harmonica trap at different temperatures $t=0.5,0.6,0.7$. (b) Condensate fraction of interacting Bose gas in harmonic trap at different temperatures $t=0.4,0.5,0.6$, for $c_{0}^{*}=0.1$ case.

at sufficiently large field when the quadratic Zeeman effect dominates and the $m_{F}=0$ atoms become condensed instead.

For simplicity, in our calculations all the energy parameters, such as $k_{B} T$ and $c_{0} N$, are rescaled by a factor $k_{B} T^{*}=\hbar\left[\sqrt{\pi / 2} \omega_{x} \omega_{y} \omega_{z} N\right]^{1 / 3}=[3 \zeta(3) \sqrt{\pi / 2}]^{1 / 3} k_{B} T_{c}^{0}$ where $T_{c}^{0}=\hbar\left(\omega_{x} \omega_{y} \omega_{z} N\right)^{1 / 3} /\left[k_{B}(3 \zeta(3))^{1 / 3}\right]$ is the BEC temperature for $N$ trapped free spin-1 atoms without external magnetic field. Then the reduced temperature reads $t=T / T^{*}=[3 \zeta(3) \sqrt{\pi / 2}]^{-1 / 3} T / T_{c}^{0}$ and $t_{c}^{0}=$ $[3 \zeta(3) \sqrt{\pi / 2}]^{-1 / 3} \approx 0.6048$ for the $c_{0}=h=0$ case.

The BEC temperature can be obtained selfconsistently from Eqs. (16) and (17), as shown in Fig. 5. Obviously, the reentrance phenomenon sustains in the presence of the trapping potentials. The $c_{0}$ term results in decrease of the BEC temperature at all magnetic fields. As studied previously, interactions tend to suppress the BEC in scalar bosons [30] and the BEC temperature drops linearly with the interaction strength $c_{0}$ when $c_{0}$ is small enough. We estimate that the linear relationship also holds in our case when both the resacled interaction parameter $c_{0}^{*}$ and the resacled magnetic field $h$ are smaller than 0.01 . But in Fig. [5, $c_{0}^{*}$ is up to 0.1 and thus the linear relationship becomes invalid.

At last, we calculate the condensate densities below the 
critical temperatures according to Eq. (13) (at low magnetic fields) or Eq. (14) (at high magnetic region). The densities of noncondensed atoms can still been obtained from Eqs. (16) and (17). We get that the condensate mainly exists in the center of the trap, while the noncondensed atoms mainly appear on the edge region. The condensate fractions for both the $c_{0}^{*}=0$ and 0.1 cases are plotted in Fig. 6(a) and (b) respectively. Again the reentrance phenomenon is illustrated for both cases. There is no much more substantial changes in comparison with the homogeneous case.

\section{SUMMARY}

In summary, we have investigated the influence of the QZE on the BEC temperature. In the case of free spinor Bose gas which exhibits a positive QZE $(q>0)$, the BEC temperature $T_{c}$ increases with the external magnetic field $h$ first, and then begins decreasing until dropping to a minimum value. $T_{c}$ increases again in stronger $h$. Thus the system shows a reentrant phenomenon of BEC with respect $h$ in the phase diagram. Similar properties are also expected in trapped interacting Bose gases. We suggest that this phenomenon can be well understood by the competition between the linear and quadratic Zeeman effects. We have also calculated the BEC temperature in the case of negative QZE $(q<0)$ and in the presence of interactions.

Acknowledgement This work is supported by the National Natural Science Foundation of China (Grant No. 10504002), the Fok Yin-Tung Education Foundation, China (Grant No. 101008), and the Ministry of Education of China (Grant No. NCET-05-0098). We thank Dr. Yajiang Hao for helpful discussions.
[1] D. M. Stamper-Kurn, M. R. Andrews, A. P. Chikkatur, S. Inouye, H. J. Miesner, J. Stenger, and W. Ketterle, Phys. Rev. Lett. 80, 2027 (1998).

[2] J. Stenger, S. Inouye, D. M. Stamper-Kurn, H.-J. Miesner, A. P. Chikkatur, and W. Ketterle, Nature (London) 396, 345 (1998).

[3] T.-L. Ho, Phys. Rev. Lett. 81, 742 (1998).

[4] T. Ohmi and K. Machida, J. Phys. Soc. Jpn. 67, 1822 (1998).

[5] Q. Gu, Phys. Rev. A 68, 025601 (2003).

[6] S. Yi, L. You, and H. Pu, Phys. Rev. Lett. 93, 040403 (2004).

[7] D. R. Romano and E. J. V. de Passos, Phys. Rev. A 70, 043614 (2004).

[8] J. Kronjager, C. Becker, M. Brinkmann, R. Walser, P. Navez, K. Bongs, and K. Sengstock, Phys. Rev. A 72, 063619 (2005).

[9] M. S. Chang, C. D. Hamley, M. D. Barrett, J. A. Sauer, K. M. Fortier, W. Zhang, L. You, and M. S. Chapman, Phys. Rev. Lett. 92, 140403 (2004); M.-S. Chang, Q. Qin, W. Zhang, L. You, and M. S. Chapman, Nat. Phys. 1, 111 (2005).

[10] H. Schmaljohann, M. Erhard, J. Kronjager, M. Kottke, S. van Staa, L. Cacciapuoti, J. J. Arlt, K. Bongs, and K. Sengstock, Phys. Rev. Lett. 92, 040402 (2004).

[11] A. T. Black, E. Gomez, L. D. Turner, S. Jung, and P. D. Lett, Phys. Rev. Lett. 99, 070403 (2007).

[12] T. Isoshima, K. Machida, and T. Ohmi, Phys. Rev. A 60, 4857 (1999).

[13] W. Zhang, D. L. Zhou, M.-S. Chang, M. S. Chapman, and L. You, Phys. Rev. Lett. 95, 180403 (2005).

[14] L.E. Sadler, J. M. Higbie, S. R. Leslie, M. Vengalattore, and D. M. Stamper-Kurn, Nature 443, 312 (2006).

[15] Q. Gu and H. Qiu, Phys. Rev. Lett. 98, 200401 (2007).

[16] T. Mizushima, N. Kobayashi, and K. Machida, Phys.
Rev. A 70, 043613 (2004).

[17] K. Murata, H. Saito, and M. Ueda, Phys. Rev. A 75, 013607 (2007).

[18] K. Yamada, Prog. Theor. Phys. 76, 443 (1982).

[19] M. V. Simkin and E. G. D. Cohen, Phys. Rev. A 59, 1528(1999).

[20] Q. Gu and R. A. Klemm, Phys. Rev. A 68, 031604(R) (2003); Q. Gu, K. Bongs, and K. Sengstock, ibid. 70, 063609 (2004); C. Tao, P. Wang, J. Qin, and Q. Gu, Phys. Rev. B 78, 134403 (2008).

[21] K. Kis-Szabo, P. Szepfalusy, and G. Szirmai, Phys. Rev. A 72, 023617 (2005); G. Szirmai, K. Kis-Szabo, and P. Szepfalusy, Eur. Phys. J. D 36, 281 (2005); S. Ashhab, J. Low Tempt. Phys. 14051 (2005).

[22] T. Isoshima, T. Ohmi, and K. Machida, J. Phys. Soc. Jpn. 69, 3864 (2000).

[23] W. Zhang, S. Yi, and L. You, Phys. Rev. A 70, 043611 (2004).

[24] L. I. Schiff and H. Snyder, Phys. Rev. 55, 59 (1939).

[25] L. Santos, M. Fattori, J. Stuhler, and T. Pfau, Phys. Rev. A 75, 053606 (2007).

[26] T. Isoshima and S. Yip, J. Phys. Soc. Jpn. 75, 074605 (2006)

[27] H. Q. Yuan, F. M. Grosche, M. Deppe, C. Geibel, G. Sparn, and F. Steglich, Science 302, 2104 (2003).

[28] H. W. Meul, C. Rossel, M. Decroux, and O. Fischer, G. Remenyi, and A. Briggs, Phys. Rev. Lett. 53, 497 (1984).

[29] H. Kleinert, S. Schmidt, and A. Pelster, Phys. Rev. Lett. 93, 160402 (2004).

[30] S. Giorgini, L. P. Pitaevskii, and S. Stringari, J. Low Temp. Phys. 109, 309 (1997); Phys. Rev. A 54, R4633 (1996).

[31] L. Pitaevskii and S. Stringari, Bose-Einstein Condensation (Clarendon Press, Oxford, 2003), Chap 10. 